\title{
Hyperprogression after Immunotherapy: Nivolumab. Analysis of Imaging Findings Associated with Hyperprogression and Tumor Growth Kinetics
}

\author{
Rishu Singla ${ }^{1}$ Ayushi Gupta ${ }^{2} \quad$ Ullas Batra $^{3} \quad$ Arvind Chaturvedi $^{1} \quad$ Avinash Rao $^{1} \quad$ Ankush Jajodia ${ }^{1}$
}

${ }^{1}$ Department of Radiology, Rajiv Gandhi Cancer Institute and Research Centre, Delhi, India

${ }^{2}$ Data Scientist, Circle of Life Health Care, Delhi, India

${ }^{3}$ Department of Medical Oncology, Rajiv Gandhi Cancer Institute and Research Centre, Delhi, India

Indian J Radiol Imaging 2021;31:345-349.
Address for correspondence Dr. Ankush Jajodia, Department of Radiology, Rajiv Gandhi Cancer Institute and Research Centre, Rohini, Sector 5, Delhi, India (e-mail: ankushjaj@gmail.com).

\begin{abstract}
Keywords

- advanced metastatic lung cancer

- hyperprogression

- immunotherapy

- tumor growth kinetics

Introduction The increased use of new checkpoint inhibitors in cancer therapy has led to the discovery of new unconventional responses, like pseudoprogression and hyperprogression disease (HPD). The study documents imaging findings of HPD and analyzes the growth kinetics in advanced metastatic cancers patients treated with immunotherapy.

Methods We retrospectively reviewed patients treated with anti-PD-1 (anti-progressive disease-1) antibody therapy (nivolumab) between January 2017 and December 2017 at our institute. The patients who exhibited early and rapid progression rates after initiation of immunotherapy were further analyzed for tumor growth kinetics (TGKs) and imaging findings. All prebaseline, baseline, and post nivolumab imaging were retrospectively reviewed to assess the TGKs, time to treatment failure (TTF), and rate of progression according to Response Evaluation Criteria in Solid Tumors (RECIST) 1.1.

Results Four patients with HPD had peculiar imaging appearance of unilateral circumferential nodular pleural thickening along with conglomerate pleural masses and effusions. Both primary and secondary sites progressed along with the appearance of new lesions in all these patients. The mean progression-free survival (PFS) was 32 days using Kaplan Meier analysis.

Conclusion The unique and recurring imaging pattern of disease progression in patients with HPD as reported in our case series in addition to TGK ratio, and TTF may prove to be of additional help in early identification of this unique and ghastly outcome.
\end{abstract}

\section{Background}

Nivolumab, an anti-PD-1 (anti-progressive disease-1) checkpoint inhibitor, has demonstrated efficacy in patients with advanced or metastatic non-small cell lung cancer (NSCLC), melanoma, small cell lung cancer, renal cell cancer, urothelial and head and neck cancers after failure to standard chemotherapeutic agents. Unlike treatment response after published online August 27, 2021
DOI https://doi.org/ $10.1055 / \mathrm{s}-0041-1734341$ ISSN 0971-3026
(C) 2021. Indian Radiological Association.

This is an open access article published by Thieme under the terms of the Creative Commons Attribution-NonDerivative-NonCommercial-License, permitting copying and reproduction so long as the original work is given appropriate credit. Contents may not be used for commercial purposes, or adapted, remixed, transformed or built upon. (https://creativecommons.org/licenses/by-nc-nd/4.0/). Thieme Medical and Scientific Publishers Private Ltd. A-12, Second Floor, Sector -2, NOIDA -201301, India 
traditional cytotoxic chemotherapy, treatment response after immunotherapy can be associated with an initial delay in response to treatment, including the appearance of new or enlarging tumors immediately after treatment that eventually resolve or decrease in size over time, without further treatment (pseudoprogression). However, there is another kind of novel response doing the rounds across oncology society these days, "hyperprogression." Hyperprogressive disease (HPD) after immunotherapy treatment is a known clinical entity in the present era. A recent multicenter, retrospective analysis of 242 patients with advanced NSCLC found that $16 \%$ of patients developed hyperprogression during anti-PD-1/PD-Ll treatment. ${ }^{1}$ The present case series is to analyze the growth kinetics and imaging findings of HPD in patients with advanced metastatic lung and renal cancers who received immunotherapy.

\section{Definition of Hyperprogression}

Champiat et $\mathrm{al}^{2}$ described HPD as a Response Evaluation Criteria in Solid Tumors (RECIST) progression at the first evaluation and a twofold or greater increase in tumor growth rate from baseline (before treatment with immunotherapy). ${ }^{2}$ Similarly, Saâda Bouzid et $\mathrm{al}^{3}$ defined hyperprogression as tumor growth kinetics (TGKs) ratio equal to or greater than 2 in patients with head and neck cancer on immunotherapy. Kato et al used a three-point definition of hyperprogression ${ }^{4}$ :

1. Time-to-treatment failure (TTF) of less than 2 months.

2. Greater than $50 \%$ increase in tumor burden compared with preimmunotherapy imaging.

3. Greater than or equal to a twofold increase in progression pace.

\section{Methods}

This is a retrospective study and was approved by the local ethics committee of Rajiv Gandhi Cancer Institute \& Research Centre. Owing to the retrospective nature of the study, informed consent from patients was not required. We describe four patients with stage IV lung and renal cancer who received nivolumab immunotherapy and showed hyperprogression (-Table 1). All but one patient was advanced metastatic lung cancer who progressed on previous multiple lines of chemotherapy.

Patients treated with monotherapy by anti-PD-1/PD-Ll antibodies, i.e., nivolumab between January 2016 and December 2017, were examined. Amongst them, ones who exhibited early and rapid progression rates after the initiation of nivolumab were studied and analyzed. All the CT scans were independently reviewed by two senior radiologists. Patients with prebaseline CT scans were included to assess TGR before starting nivolumab. All prebaseline, baseline, and post nivolumab imaging were retrospectively reviewed to assess the TGKs, TTF, and increase in progression pace according to RECIST 1.1 .

The following data were also recorded: age, gender, PD-L1 analysis, patterns of hyperprogression, date of start of nivolumab, and date of last follow-up or adverse event such as death.

$T_{\text {PRE }}, T_{0}$, and $T_{\text {POST }}$ stand for the time of prebaseline, baseline, and first imaging, respectively. $S_{\text {PRE }}, S_{0}, S_{\text {POST }}$ stand for the sum of the largest diameter of target lesions at prebaseline, baseline, and first imaging, respectively. $\mathrm{TGK}_{\mathrm{PRE}}$ was defined as the difference of the sum of the largest diameters of the target lesions (according to RECIST) per unit of time between prebaseline and baseline imaging: $\left(S_{0}-S_{\text {PRE }}\right) /\left(T_{0}-T_{\text {PRE }}\right)$. Similarly, TGK $_{\text {POST }}$ was defined as: $\left(S_{\text {POST }}-S_{0}\right) /\left(T_{\text {POST }}-T_{0}\right)$.

The TGK ratio $\left(\mathrm{TGK}_{\mathrm{R}}\right)$ was defined as the ratio of $\mathrm{TGK}_{\mathrm{POST}}$ to $\mathrm{TGK}_{\mathrm{PRE}} \cdot \mathrm{TGK}_{\mathrm{R}}>1$ indicated tumor growth acceleration, whereas $0<\mathrm{TGK}_{\mathrm{R}}<1$ and $\mathrm{TGK}_{\mathrm{R}}<0$ indicate tumor deceleration and tumor shrinkage, respectively. Hyperprogression was defined as $\operatorname{TGK}_{\mathrm{R}} \geq 2$.

\section{Statistical Analysis}

Descriptive statistics is presented with line graphs. $\mathrm{TGK}_{\mathrm{R}}$ was calculated using the formula given in the assessment section. Kaplan Meier analysis was performed for progression-free survival (PFS). Median PFS with a 95\% confidence interval was reported.

\section{Results}

There were four patients with advanced metastatic lung/renal cancer treated with monotherapy by anti-PD-1/PD-L1 antibodies in this case-series. - Table 1 shows the demographic and clinical details of these four patients. - Fig. 1 represents diameter growth according to RECIST 1.1 criteria. The sum of the diameter shows an exponential increase after treatment with nivolumab (-Figs. 1 and 2 ).

Three out of four (75\%) patients showed accelerated growth measured through TGKs (Cases\# 1,2 and 4 TGK >2) (-Figs 3-6). Case\#3 also developed rapid worsening of symptoms after two cycles of nivolumab monotherapy. The $\mathrm{X}$-ray chest showed marked increase in air space opacities and pleural effusion. The patient also developed anterior wall myocardial infarction, and hence cross-sectional imaging could not be performed at the time of onset of symptoms due to his poor general condition. Hence TGK recorded is less than 2 for case 3 . However, the increase in tumor burden was $>50 \%$ as compared with prebaseline imaging, and TTF was less than 2 months.

Median progression-free $(95 \% \mathrm{CI})$ survival was 32 (21.242.8) days (-Fig. 3 ).

Contrast-enhanced computed tomography chest findings were notably similar in all of them. There was a development of unilateral circumferential pleural nodularity with conglomerate pleural masses and associated pleural effusion. The laterality corresponded to the side of primary baseline disease.

\section{Discussion}

In our series, all but one patient experienced hyperprogression as defined by $\mathrm{TG}_{\mathrm{KR}}>2$ after anti-PD-1/PD-Ll therapy. We 
Table 1 Clinical characteristics of patients

\begin{tabular}{|c|c|c|c|c|}
\hline & Case 1 & Case 2 & Case 3 & Case 4 \\
\hline Age & $62 y$ & $56 y$ & $46 y$ & $62 y$ \\
\hline Gender & Male & Male & Male & Male \\
\hline Primary diagnosis & $\mathrm{mRCC}$ & $\mathrm{mNSCLC}$ & $\mathrm{mNSCLC}$ & $\mathrm{mNSCLC}$ \\
\hline Date of detection & May 2016 & July 2016 & April 2013 & July 2017 \\
\hline EGFR/ALK mutation status & Not done & Negative & EGFR mutant & Negative \\
\hline Anti PD-1, PD-L1 status & Not done & $<1 \%$ & $<1 \%$ & $<1 \%$ \\
\hline \multirow[t]{3}{*}{ Treatment history } & \multirow[t]{2}{*}{$\begin{array}{l}\text { Surgery for both primary and } \\
\text { metastatic site followed by }\end{array}$} & \multirow{2}{*}{$\begin{array}{l}\text { CT/RT till } \\
\text { November } 16 . \\
\text { PD in May } 17 . \\
\text { Palliative CT. }\end{array}$} & \multirow{2}{*}{$\begin{array}{l}\text { Multiple lines of CT } \\
\text { till June 2015-PD, } \\
\text { Gefitinib/. }\end{array}$} & \multirow[t]{3}{*}{$\begin{array}{l}\text { Three cycles of chemo- } \\
\text { therapy. PD }\end{array}$} \\
\hline & & & & \\
\hline & Sutent & $\begin{array}{l}\text { Six cycles till } \\
\text { September 17. PD } \\
\text { in October } 2017 .\end{array}$ & $\begin{array}{l}\text { Erlotinib till } \\
\text { September 16-PD. }\end{array}$ & \\
\hline $\begin{array}{l}\text { Duration of treatment before } \\
\text { progression }\end{array}$ & $6 \mathrm{mo}$ & 1 y 3 mo & 3 y $5 \mathrm{mo}$ & $3 \mathrm{mo}$ \\
\hline CT findings (Baseline) & $\begin{array}{l}\text { Bilateral lung lesions, mediasti- } \\
\text { nal lymphadenopathy, bilateral } \\
\text { pleural effusions, pericardial } \\
\text { effusion, bone lesions. }\end{array}$ & $\begin{array}{l}\text { Left hilar mass, } \\
\text { right lung nod- } \\
\text { ules, moderate } \\
\text { left pleural } \\
\text { effusion. }\end{array}$ & $\begin{array}{l}\text { Left lung upper lobe } \\
\text { mass, bilateral lung } \\
\text { nodules, mediastinal, } \\
\text { and left lower cervical } \\
\text { lymph nodes. }\end{array}$ & $\begin{array}{l}\text { Right lung mass with } \\
\text { mediastinal lymph } \\
\text { nodes and left adrenal } \\
\text { metastasis. }\end{array}$ \\
\hline \multirow{3}{*}{$\begin{array}{l}\text { Date of start of nivolumab } \\
\text { duration of immunotherapy } \\
\text { CT findings (Post nivolumab). }\end{array}$} & January 18, 2017 & October 12, 2017 & September 24, 2016 & October 12, 2017 \\
\hline & Two cycles & One cycle & Four cycles & One cycle \\
\hline & $\begin{array}{l}\text { >twofold increase in the size } \\
\text { of lung lesions, new circum- } \\
\text { ferential left-sided nodular } \\
\text { pleural thickening with a } 150 \% \\
\text { increase in the size of mediasti- } \\
\text { nal lymph nodes. }\end{array}$ & $\begin{array}{l}\text { Progressive left } \\
\text { lung mass with } \\
\text { new left-sided } \\
\text { nodular pleural } \\
\text { thickening and } \\
\text { minimal right } \\
\text { pleural effusion. }\end{array}$ & $\begin{array}{l}\text { Progressive left lung } \\
\text { mass and bilateral } \\
\text { lung nodules, pro- } \\
\text { gressive mediastinal } \\
\text { lymph nodes, new } \\
\text { circumferential left } \\
\text { pleural deposits, new } \\
\text { brain, liver, adrenal, } \\
\text { and bone lesions. }\end{array}$ & $\begin{array}{l}\text { Progressive right hilar } \\
\text { lung mass, new bilat- } \\
\text { eral pleural effusion, } \\
\text { diffuse nodular circum- } \\
\text { ferential right pleural } \\
\text { thickening and nodular } \\
\text { deposits, progressive } \\
\text { mediastinal lymph } \\
\text { nodes. }\end{array}$ \\
\hline Last follow-up & LFU & LFU & LFU & $\begin{array}{l}\text { Died of cardiac arrest in } \\
\text { November } 2017\end{array}$ \\
\hline
\end{tabular}

Abbreviations: ALK, anaplastic lymphoma kinase; CT, computed tomography; EGFR, epidermal growth factor receptor; LFU, lost to follow-up; NSCLC, non-small cell lung cancer; PD, progressive disease.

Note: This is an open access journal, and articles are distributed under the terms of the Creative Commons Attribution-NonCommercial-ShareAlike 4.0 License, which allows others to remix, tweak, and build upon the work noncommercially, as long as appropriate credit is given, and the new creations are licensed under the identical terms.

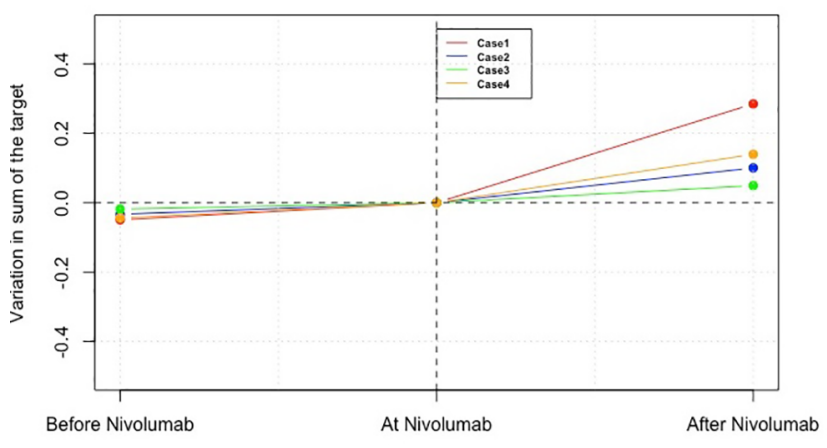

Fig. 1 Diameter growth before and after nivolumab.

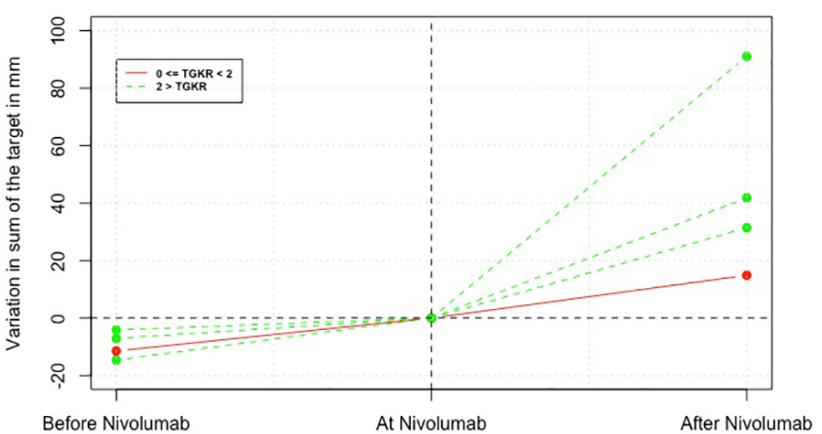

Fig. 2 TGKs before and after. TGK, tumor growth kinetics. 

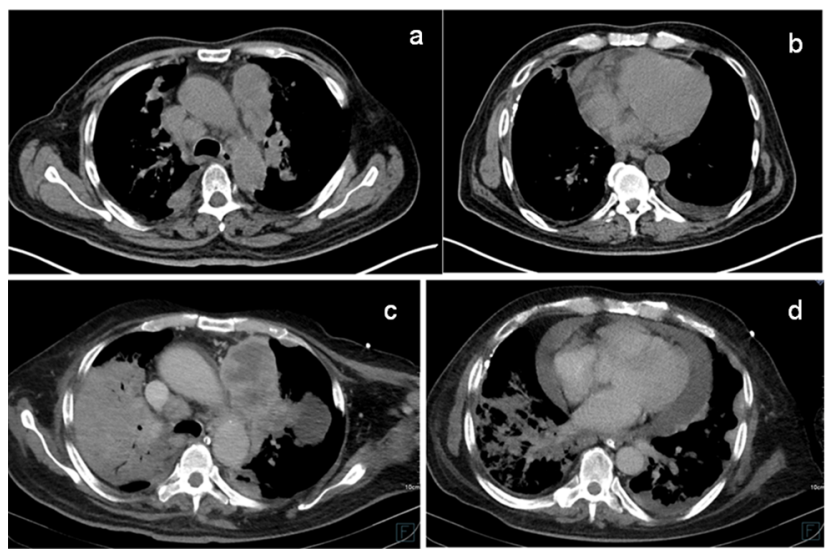

Fig. 3 CT images obtained pre (a and $\mathbf{b}$ ) and post (c and $\mathbf{d}$ ) nivolumab therapy in Case 1: Lung lesions show an increase in size. Bilateral pleural effusion/nodular lesions and pericardial effusion are new developments. CT, computed tomography.

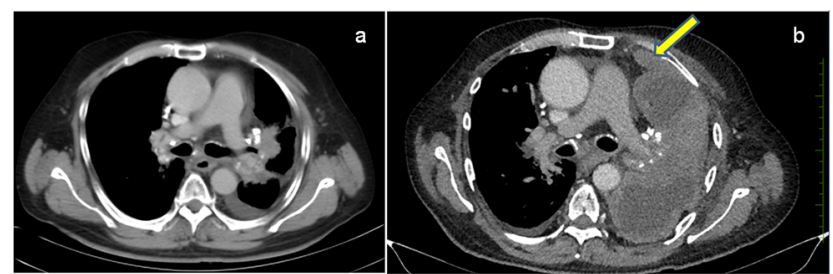

Fig. 4 (a and $\mathbf{b}$ ) Labeled contrast CT axial obtained pre and post nivolumab therapy in Case 2: nodular pleural lesions show an increase in size (arrows), supporting the hypothesis of increase pleural nodular pattern of hyperprogression on nivolumab therapy. CT, computed tomography.
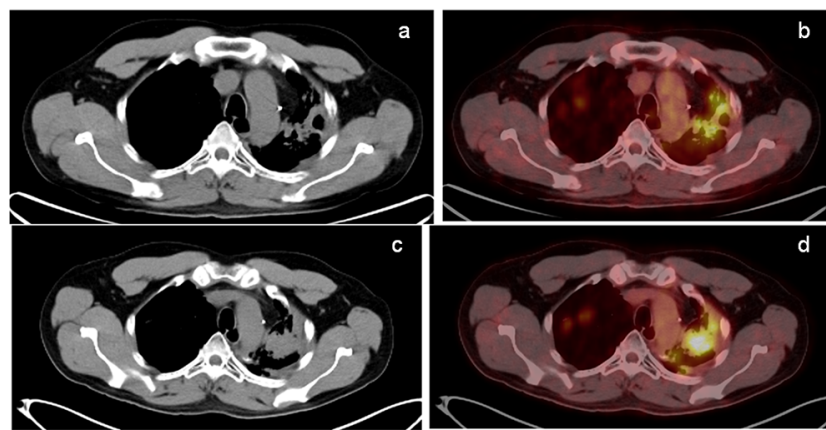

Fig. 5 Labeled PET-CT fusion images in reconstructed formats obtained pre ( $\mathbf{a}$ and $\mathbf{b}$ ) and post (c and $\mathbf{d}$ ) nivolumab therapy in Case 3: left lung mass and lung nodules show an increase in extent and metabolic activity. PET-CT, positron emission tomography-computed tomography.
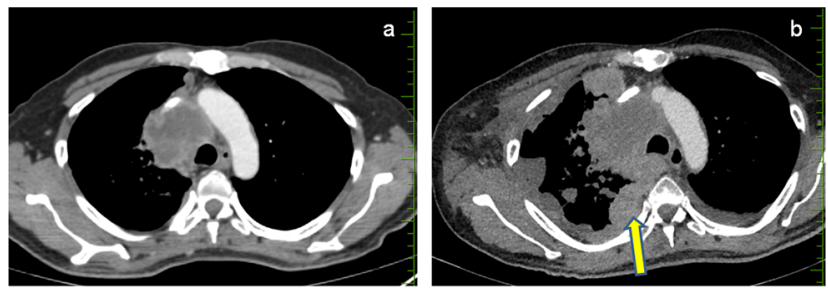

Fig. 6 Labeled contrast CT axial obtained pre (a) and post (b) nivolumab therapy in Case 4: nodular pleural lesions show an increase in size (arrows), supporting the hypothesis of increase pleural nodular pattern of hyperprogression on nivolumab therapy. CT, computed tomography. could not perform CT chest imaging of one patient when he developed worsening of symptoms leading to discontinuation of treatment. This led to an increase in the time duration between two assessment scans, i.e., $T_{\text {post }}-T_{0}$ and a decrease in TGK $_{R}$ to less than 2. However, TTF was $<2$ months in all four patients in accord with the definition given by Kato et al. ${ }^{4}$ Also, there was $>50 \%$ increase in tumor burden at the time of next imaging compared with preimmunotherapy imaging fulfilling one of the criteria of HPD. But it is important to note here that the patient was not under any kind of anticancer treatment during this period making this observation suboptimal.

Hyperprogression was associated with poor prognosis in our study, in accord with other published reports. ${ }^{1,2}$ HPD was associated with short PFS (32 days) in our series. We could not calculate overall survival as three out of four patients lost to follow-up.

Kato et $\mathrm{al}^{4}$ investigated potential genomic markers associated with hyperprogression after immunotherapy. They reported TTF $<2$ months in six of 155 patients and TGK $>2$ and an increase in tumor burden of $>50 \%$ in four out 155 patients. They also suggested MDM 2/MDM 4 and epidermal growth factor receptor (EGFR) alterations correlated with TTF $<2$ months. We could not establish any correlation between EGFR aberrations in our case series and hyperprogressors. Two out of four patients were EGFR/anaplastic lymphoma kinase (ALK)-negative. One patient was EGFR mutant and EGFR/ALK analysis was not performed in the patient of metastatic renal cell cancer.

Champiat et $\mathrm{al}^{2}$ demonstrated that $9 \%$ of patients (12/131) showed a >twofold increase in tumor growth in their study. They observed that HPD was associated with older age and with worse overall survival. They observed no correlation between hyperprogression and baseline tumor burden and the number of previous lines of chemotherapies. Twenty-seven percent of their patients underwent PDL- 1 testing and they did not find any difference of PD-L1 status between HPD and other patients.

All our patients underwent anti-PD-Ll testing and were negative. The role of PD-L1 expression/amplification is known in predicting response to immunotherapy ${ }^{5}$ but no correlation has been established between HPD and anti-PDL-1 status till date.

We also describe the pattern of hyperprogression in our case series, which has not been described in any previous study. The imaging pattern observed was independent of primary tumor type and we recorded similar findings in all the four cases such as unilateral diffuse pleural nodularity with loss of ipsilateral hemithoracic volume. The pleural nodular thickening was circumferential and extensive forming conglomerate large-sized pleural masses. None of these patients had pleural disease at the beginning of treatment. Unilateral circumferential nodular pleural thickening was a key finding and was seen on the side of primary lung mass. This finding was unique with immunotherapy as these patients also previously received multiple lines of chemotherapy and showed no such imaging features. These patients also developed either new or increase in pleural effusion after immunotherapy. All 
patients showed progression at both primary and secondary sites. The imaging findings correlated well with the severity of patient's symptoms.

Our series is unique as no previous study has described these imaging findings.

Immunotherapy can also lead to unconventional responses such as pseudoprogression. Pseudoprogression is radiological progression followed by a response on subsequent imaging on sustained immunotherapy. It is important to differentiate between the two as hyperprogression calls for immediate discontinuation of treatment, whereas treatment is continued for pseudoprogression exhibiting clinical response. As mentioned earlier, hyperprogression can be measured by assessing $\mathrm{TGK}_{\mathrm{R}}$, tumor burden, and TTF. The chief factor before discontinuing treatment is clinical status. The HPD patients show significant clinical deterioration, whereas pseudoprogression is considered when a patient is showing a clinical benefit.

Our study has several limitations. Its biggest limitation is the small size. The second limitation is that we used RECIST 1.1 criteria which are now replaced by iRECIST criteria for response evaluation after immunotherapy. These criteria are different from RECIST1.1 in that radiologically progressive disease needs to be confirmed by a follow-up examination in case of clinical response. ${ }^{6,7}$ All our patients showed severe clinical worsening, and hence no confirmatory scans were performed.

Also, in contrast to RECIST 1.1, the summed diameters of new lesions appearing on follow-up examinations are recorded separately (maximum two per organ, five overall) for iRECIST and not included in the sum of the tumor burden at baseline or time of nadir. Using RECIST 1.1 we did not record the diameter of new lesions that appeared at time point $T_{0}$ and hence could not assess their contribution to tumor burden at time point $T^{\text {post }}$.

However, it is also noteworthy to mention that most of the definitions of HPD suggested by various authors used RECIST 1.1 criteria., ${ }^{2,8}$ Presently, iRECIST criteria do not have provisions to recognize hyperprogression or differentiate hyperprogression from pseudoprogression. In view of this limitation, the early identification of HPD becomes even more important as it warrants cessation of treatment in contrast to immune unconfirmed progressive disease, which ensues treatment beyond progression.

\section{Conclusion}

The unique and recurring imaging pattern of disease progression in patients with HPD as reported in our case series in addition to $\mathrm{TGK}_{\mathrm{R}}$, and TTF may prove to be of additional help in early identification of this unique and mostly lethal treatment outcome. The radiologist may have a key role in alerting medical oncologists to potential risks of these new treatments. Further large patient cohort studies are needed to explore the imaging patterns of hyperprogression and to recognize genomic alterations and imaging predictors associated with HPD.

\section{Financial Support and Sponsorship \\ None.}

\section{Conflict of Interest}

None declared.

\section{References}

1 Ferrara R, Caramella C, Texier M, et al. Hyperprogressive disease (HPD) is frequent in non-small cell lung cancer (NSCLC) patients (pts) treated with anti PD1/PD-L1 monoclonal antibodies (IO. Ann Oncol 2017;28:v464-v465

2 Champiat S, Dercle L, Ammari S, et al. Hyperprogressive disease (HPD) is a new pattern of progression in cancer patients treated by anti-PD-1/ PD-L1. Clin Cancer Res 2017;23(8):1920-1928

3 Saâda-Bouzid E, Defaucheux C, Karabajakian A, et al. Hyperprogression during anti-PD-1/PD-L1 therapy in patients with recurrent and/or metastatic head and neck squamous cell carcinoma. Ann Oncol 2017;28(7):1605-1611

4 Kato S, Goodman A, Walavalkar V, Barkauskas DA, Sharabi A, Kurzrock R. Hyperprogressors after immunotherapy: analysis of genomic alterations associated with accelerated growth rate. Clin Cancer Res 2017;23(15):4242-4250

5 Patel SP, Kurzrock R. PD-L1 expression as a predictive biomarker in cancer immunotherapy. Mol Cancer Ther 2015;14(4):847-856

6 Therasse P, Arbuck SG, Eisenhauer EA, et al. New guidelines to evaluate the response to treatment in solid tumors. European Organization for Research and Treatment of Cancer, National Cancer Institute of the United States, National Cancer Institute of Canada. J Natl Cancer Inst 2000;92(3):205-216

7 Eisenhauer EA, Therasse P, Bogaerts J, et al. New response evaluation criteria in solid tumours: revised RECIST guideline (version 1.1. Eur J Cancer 2009;45(2):228-247

8 Ferrara R, Mezquita L, Texier M, et al. Hyperprogressive disease in patients with advanced non-small cell lung cancer treated with PD-1/PD-L1 inhibitors or with single-agent chemotherapy. JAMA Oncol 2018;4(11):1543-1552 